\title{
The Current State of Dental Sleep Medicine Practice in Academic Institutions: A Questionnaire-Based Study
}

\author{
Nader Karimi, DDS, MS ${ }^{1}$; Noshir Mehta, BDS, MDS, DMD, MS ${ }^{1}$; Sarah E. Pagni, PhD, MPH ${ }^{1}$; Ekaterini Antonellou, DMD, MS ${ }^{1}$; \\ Eileen H. Doherty, $\mathrm{MS}^{1}$; Leopoldo P. Correa, BDS, MS ${ }^{1}$
}

${ }^{1}$ Tufts University School of Dental Medicine, Boston, Massachusetts

\begin{abstract}
Study Objectives: The aim of this study is to evaluate the current state of dental sleep medicine practice at the United States and Canada academic institutions.

Methods: An electronic survey created in Qualtrics was sent via email. The survey was sent to the 76 dental schools academic institute in the United States and Canada, 16 of which were known to have a dental sleep medicine clinic. The survey was emailed to the program directors of the sleep medicine clinics at these dental schools. For the remaining 60 schools that do not have a dental sleep medicine program, the survey was instead sent to the academic dean. The multiple choice 21 -item survey was validated and received Institutional Review Board approval.

Results: The response rate was 37\% (28 schools), of which $86 \%$ were in the United States. Of the respondents, $45 \%$ ( 12 schools) had a dental sleep medicine clinic. The dental sleep medicine clinics were either an independent/ part of diagnostic services (42\%) or incorporated with other departments. Almost $33 \%$ of the schools that have a sleep medicine clinic reported difficulty integrating sleep medicine into other existing programs. Eighty-three percent of respondents reported the presence of one to two board certified dental sleep medicine faculty members, whereas the remaining schools did not have any.

Conclusions: This current study indicates that dental sleep medicine in academic institutions is faced with many obstacles that need to be addressed. Dental sleep medicine is a growing field that needs more attention from educational institutes. Also, the positive effect of having board-certified dental sleep medicine faculty should be noted.

Keywords: dental sleep medicine education, practice of dental sleep medicine in academia, dental sleep medicine practice questionnaire based study, dental sleep medicine in North America, sleep in dentistry sleep education in dental schools

Citation: Karimi N, Mehta N, Pagni SE, Antonellou E, Doherty EH, Correa LP. The current state of dental sleep medicine practice in academic institutions: A questionnaire-based study. J Dent Sleep Med. 2019;6(4)
\end{abstract}

\section{INTRODUCTION}

Sleep disorders, such as obstructive sleep apnea (OSA), have a deleterious effect on quality of life, not to mention sleep. OSA, a common sleep disorder, is characterized by the blockage of airflow during sleep and includes snoring and daytime sleepiness as its most common symptoms. Several studies indicate that lack of sleep can lead to the development of cardiovascular disease and diabetes by altering the hormonal balance, glucose regulation, and increased inflammatory markers and blood pressure. ${ }^{1-3}$ Primary care physicians are keen to screen, identify and properly refer patients with OSA. Previous studies have focused largely on the physician's education and attitude regarding sleep disorders. ${ }^{4-9}$

Sleep-related disorders have also been consistently associated with periodontal disease. ${ }^{10,11}$ OSA has also been linked to cardiovascular disorders such as coronary artery disease, stroke, heart attacks, congestive heart failure, atrial fibrillation, ventricular arrhythmias, impaired quality of life, and increased mortality. Additionally, there is an association between increased lack of sleep and increased incidence of motor vehicle accident. ${ }^{12-19}$

The field of somnology/sleep medicine, however, is minimally discussed in dental schools, particularly at the predoctoral level..$^{20}$ An editorial from 2006 titled "Sleep Disorders and Sleep Deprivation: An Unmet Public Health Problem" reported that an estimated 50 to 70 million Americans have some type of sleep disorder and that without treatment these disorders pose a significant public health threat. $^{21}$

Current gaps in education and research regarding disorders of sleep and wakefulness can be playing an underrecognized role in the rise of metabolic disorders, contributing to a higher economic burden for the population. In one study, the investigators concluded that strategies to increase awareness of dental sleep medicine and the importance of treating sleep-related disorders can play a significant role in advancing patient care. ${ }^{5,8}$ These strategies include incorporating interdisciplinary dental sleep programs in academic hospitals, establishing a clinical network for sleep medicine research, and starting new research training programs across the spectrum of somnology/sleep medicine.

Results from a survey show the current level of medical education as it relates to sleep and sleep disorders in New Zealand and Australian medical schools are somewhat lacking. Surveyed schools reported that an average undergraduate time of 4.5 hours was devoted to dental sleep medicine and appeared to be concentrated in the fifth 
year of school; 2 out of 6 schools included dental sleep medicine in the second year with a mean of only 0.33 hours. ${ }^{22}$

Screening patients for OSA and other sleep-related breathing disorders (SRBDs) is an important aspect of the care offered by dental professionals. Dental professionals can play a pivotal role in reducing OSA prevalence, resulting in possibly reducing patient morbidity and mortality. This may be accomplished by routinely screening and treating patients for SRBDs; however, newer dental students receive a less-than-adequate level of education regarding screening importance. ${ }^{23}$ Dental schools require a greater level of sleep education than what is currently available because of the ability of disorders to drive periodontal disease, cardiovascular disease, and other metabolic and endocrinologic health issues. ${ }^{24-29}$

A retrospective, 2-year follow-up study by Ivanoff and Pancratz examined whether screening protocols (as well as education on the importance of screening) had improved at a US dental school since 2011.25 Although screening did improve over a 2-year period, there were still some areas of screening that require further refinement. Their study highlights the fact that education regarding the screening and management of sleep disorders should be incorporated throughout predoctoral and postdoctoral training to a greater degree than it is currently to advance patient care. $^{14}$

The role of dental sleep medicine in the educational curriculum of dental schools has been scrutinized more closely these past few years, thanks in part to the increased awareness of sleep disorders and their association with oral health. Although education on SRBDs has improved in dental schools, the level at which it can help future dentists effectively treat patients with these disorders remains up for debate. ${ }^{1}$

A lack of medical education regarding sleep disorders is not just specific to the United States. The nature and extent of sleep medicine education provided by medical schools were investigated by many researchers. The results indicated that medical students and primary care physicians do not have the necessary knowledge and clinical skills regarding sleep disorders management. Inadequate curricular hours, resources, teaching staff, and facilities might be the causes of such deficiency. ${ }^{23,24}$ A study by Talaat et al. ${ }^{26}$ found that an average of 1.2 hours was devoted to dental sleep medicine in the Middle East medical schools, a time not efficient enough to train future physicians to screen and treat patients with sleep-related disorders. Considering that these disorders are relatively high in this population group, incorporating further education measures in these schools is crucial for reducing patient morbidity in Middle Eastern countries.

In 2003, Ivanhoe et al. ${ }^{30}$ reported that 18 of 43 dental educational facilities in the United States confirmed that they teach about and manage upper airway disorders. For those 18 schools, teaching hours averaged to 2.5 . It is worth noting that 25 of the 43 dental schools (58\%) stated that there is no time to teach about sleep disorders. Additionally, merely 6 of the 18 schools reported teaching dental sleep medicine to the predoctoral students, 5 of 18 at taught sleep medicine to the postdoctoral students, and the remaining 7 dental schools taught sleep medicine to both predoctoral and postdoctoral students.

In general, predoctoral dental students are not sufficiently trained to in the diagnosis and management of OSA. ${ }^{31,32}$ Numerous reasons exist as to why there is currently an absence, or a severe deficiency, in dental sleep medicine practice at academic institutions. Challenges exist, such as achieving a multidisciplinary approach and collaboration with sleep physicians or similar work within the same institution by faculty members and may require significant improvement of institutions' organizational structure. $^{33-35}$

Strategies to improve the level of dental sleep medicine practice at the academic institutions may include incorporating dental sleep medicine education into the curricula, motivating and supporting faculty members to develop an interdisciplinary approach between departments within their own dental schools, and establishing a clinical network with sleep centers and physicians to potentially incorporate a dental sleep medicine clinic in their institutions. $^{5}$

Therefore, we seek to examine dental sleep medicine practice at the academic institution level and to determine whether current practice is sufficient to meet the needs of both dental professionals and patients. Additionally, we will evaluate the level of dental sleep medicine education and research at current institutions. The significance of this survey study was to identify the challenges encountered in the development of dental sleep medicine practice in academia. This will serve to provide strategies and potentially protocols for the implementation of dental sleep medicine practice in more dental schools.

Potentially, this study may provide information on the areas of improvement needed as well as assist in the development and implementation of greater dental sleep medicine practices at academic institutions.

\section{METHODS}

A total of 76 US and Canada dental schools were surveyed in this study. An electronic survey was utilized and send via email. To the 16 schools known to have a dental sleep clinic, the email was sent to the director or faculty member of the dental sleep clinic. The academic dean was approached with the survey in the remaining 60 schools that did not have a dental sleep clinic. Qualtrics was used for sending the electronic survey via email. The survey had 21 multiple- choice items, and some items required an optional written response. 
Descriptive statistics (counts and percentages) were calculated. SPSS version 24 was used (Armonk, NY: IBM Corp).

\section{RESULTS}

Thirty-seven percent (28/76) of dental schools surveyed in North America responded to this survey. Of the dental schools responding to the survey, $86 \%$ (24/28) were in the United States. Among the $86 \%$ of dental schools $(24 / 28)$ in the United States, $4(16.67 \%)$ were from Northeast region, 5 (20.83\%) from Southeast, 4 (16.67\%) from Midwest, 5 (20.83\%) from Southwest, and 6 (25\%) from the Western region. Seventy-five percent of respondents $(21 / 28)$ were affiliated with the public university and $25 \%$ (7/28) with a private university.

Twenty-seven survey responders answered whether their institution had a dental sleep medicine clinic. Among the respondents, $45 \%(12 / 27)$ had a dental sleep medicine clinic at their institution whereas $55 \%(15 / 27)$ did not. The dental sleep medicine clinics were incorporated in the following departments: orofacial pain and temporomandibular disorder, $42 \%$ (5/12); orthodontics, $8 \%$ (1/12); and oral medicine, $8 \%$ (1/12). In addition, $42 \%(5 / 12)$ stated that dental sleep medicine clinics were either independent of any department or part of diagnostic services, facial pain and dental sleep clinic, advanced care center, or department of diagnostic sciences (Figure 1).

Forty-two percent of respondents (5/12) did not report any challenges with implementing the dental sleep medicine program at their institution, whereas $25 \%$ (3/12) stated not having a sufficient number of faculty members trained in dental sleep medicine. Other challenges stated by $33 \%$ (4/12) included difficulty integrating into existing programs, lack of administrative support or space for the clinic, and staffing and billing issues (Figure 2). Eightythree percent of respondents (10/12) reported one to two board certified dental sleep medicine faculty members whereas $17 \%(2 / 12)$ stated the presence of no board certified dental sleep medicine faculty members at their institution (Figure 3).

Of the 12 institutions with dental sleep medicine clinic, $50 \%(6 / 12)$ stated that only faculty members treated patients and the other $50 \%(6 / 12)$ stated both faculty and residents treat patients.

Regarding the source of patient referrals to the school's sleep clinic, $83 \%$ of respondents (10/12) stated that they get their patient referrals from sleep centers. Nearly $91 \%$ of the respondents (11/12) indicated that their referrals came from sleep physicians, $75 \%$ (9/12) from within the dental school, $41 \%$ (5/12) from dentists, and $16 \%(2 / 12)$ stated their patient referral source as other including area internists, or cardiologists, ear, nose, and throat specialists, etc (Figure 4).

All 12 of the respondents stated that they use a custom fabricated adjustable oral appliance, whereas 25\% (3/12) reported using a prefabricated and adjustable oral appliance. In addition, $17 \%$ (2/12) and 33\% (4/12) used temporary and tongue retainer devices, respectively.

Regarding the type of combination therapy offered to the patients, $75 \%$ of responders $(9 / 12)$ indicated that they offer combination therapy of mandibular advancement device with either positive airway pressure (PAP) or positional therapy to their patients. Only $17 \%$ of respondents $(2 / 12)$ stated that they offer a tongue-retaining device with PAP therapy to their patients.

Regarding techniques used to minimize side effects, of the 12 respondents, $92 \%(11 / 12)$ stated that they use

Figure 1. Departments integrated with the sleep medicine clinic.

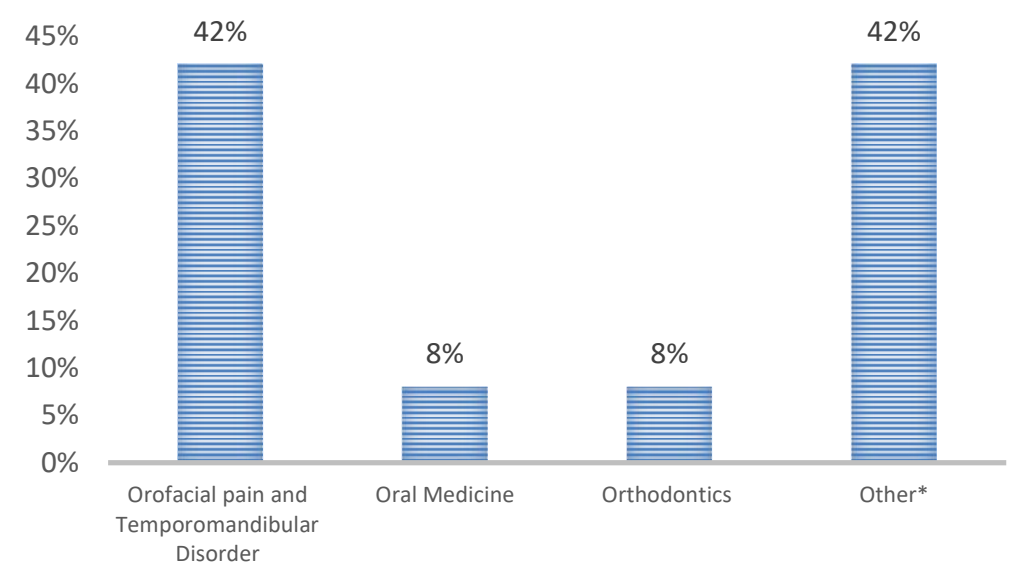

*Other: Diagnostic Services, Facial pain \& sleep clinic, Advanced Care Center, Independent of any department and Department of Diagnostic Sciences 
Figure 2. Challenges in implementing a dental sleep medicine clinic.

No challenges (5) Understaffed (3) Administrative, no space, funds (4)

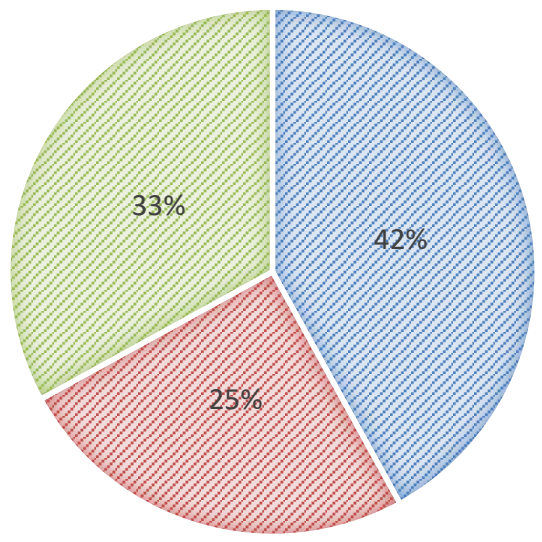

Figure 3. The presence of board certified trained dental sleep medicine faculty members.

Do not have board certified trained dental sleep medicine faculty (2)

1-2 board certified trained dental sleep medicine faculty (10)

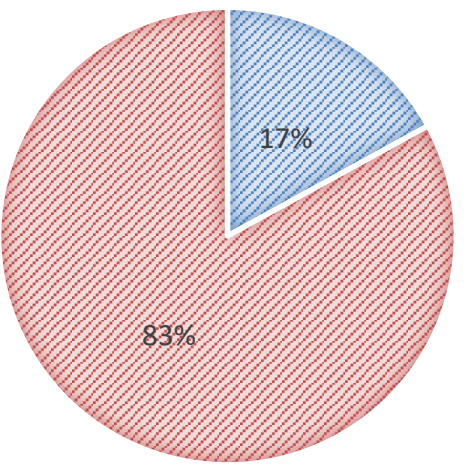

Figure 4. Patient referral sources.

틀 Source of referrals

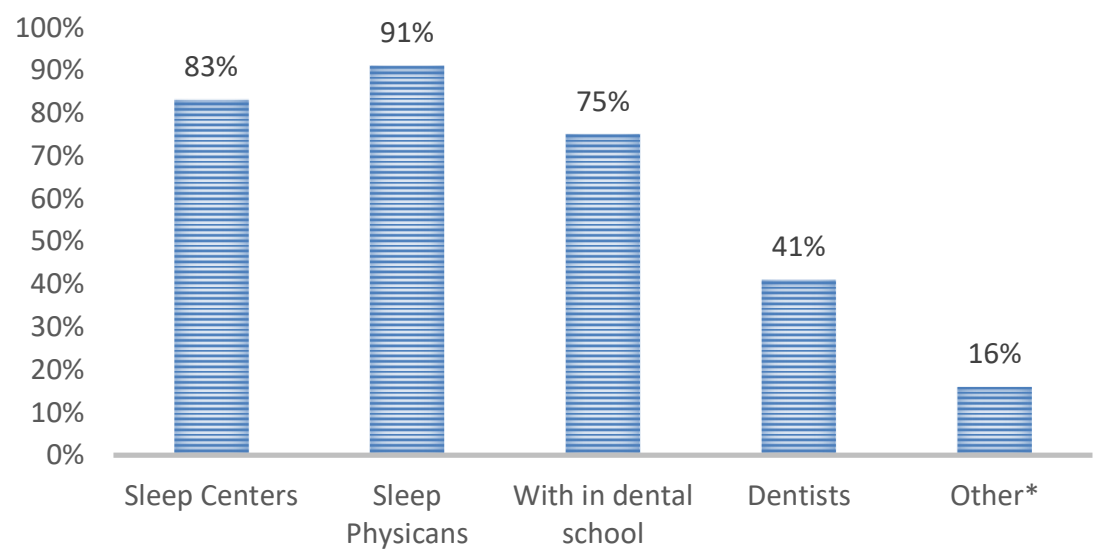

Other*: Area internists, cardiologists, ENTs, self-referred etc. 
techniques to minimize bite side effects whereas $8 \%(1 / 12)$ did not. In addition, $75 \%$ (9/12) stated that they provided the technique to minimize bite side effects at the time of sleep oral appliance delivery whereas $25 \%$ (3/12) provided the technique as needed if bite side effects developed.

Regarding their protocol schedule for short-term follow-up visits, of the 11 respondents, $36 \%$ (4/11) stated follow-up visits every 2 weeks and 36\% (4/11) stated every 4 weeks. In addition, $27 \%$ of respondents (3/11) stated other follow-up schedules including initial follow-up at 2 weeks after delivery of the device followed by every 4 weeks for the first 3 months or initial follow-up at 2 weeks after delivery of the device followed by a visit at 2 to 3 months until resolution of subjective symptoms.

Regarding the schedule for long-term follow-up visits, of the 11 respondents, $64 \%$ (7/11) stated follow-up visit at 6 months and 1 year after completion of therapy, 9\% (1/11) stated follow-up visits as needed, and $27 \%$ (3/11) stated other schedules such as every year.

Among the 11 respondents indicating how many patients are scheduled monthly, 91\% (10/11) scheduled 5 to 15 new patients monthly whereas $9 \%(1 / 11)$ scheduled 1 to 5 new patients monthly. Of the 11 responders to question $20,45 \%(5 / 11)$ stated that fewer than $10 \%$ of patients were lost to follow-up, $18 \%(2 / 11)$ stated lost to follow-up of $10 \%$ to $20 \%$ of patients, and $36 \%$ (4/11) stated lost to follow-up of $>20 \%$ patients.

Sixty-three percent of the respondents (7/11) stated that they used polysomnography to confirm treatment efficacy, 26\% (5/11) used home sleep apnea testing, 10.5\% $(2 / 11)$ assessed treatment efficacy based on patient's feedback, and 26\% (5/11) used other techniques such as cardiopulmonary coupling or referral back to the sleep physician for evaluation and possible home sleep apnea testing or polysomnography per their recommendation.

Ninety-one percent of respondents $(10 / 11)$ to the question about having a continuing education (CE) program had CE programs in dental sleep medicine at their institution whereas only $9 \%(1 / 11)$ did not have continuing education programs in dental sleep medicine (Figure 5). Of the 10 respondents, on the frequency of dental sleep CE courses, $60 \%(6 / 10)$ stated that courses were offered every 10 to 12 months, $20 \%(2 / 10)$ stated every 4 to 6 months, and $10 \%(1 / 10)$ each stated every 1 to 3 months or every 7 to 9 months (Figure 6).

Regarding whether the dental sleep CE course offered at their institution was a minimum of 25 hours. $60 \%$ of respondents (6/10) thought that the CE course was not a minimum of 25 hours whereas $40 \%$ (4/10) thought that it was a minimum of 25 hours.

\section{DISCUSSION}

Several studies have indicated that there is no systematic method to improve clinician practices and/or patient outcomes in dental sleep medicine. ${ }^{35,36}$ In the current study, only $37 \%$ of dental schools (28/76) responded to the survey and most them were located in the United States. According to the data obtained from this survey, only $45 \%$ of dental schools responding (12/28) had dental sleep medicine clinic at their institution. This finding highlights the lack of widespread dental sleep medicine practice at academic institutions.

Consistent with the findings of dental Sleep Research Society surveys of 2009 and 2012 indicating a paucity of independent structures in administrative departments of academic institutions for the practice of dental sleep medicine, the dental sleep medicine clinics were in various departments including orofacial pain and temporomandibular disorder, and orthodontics. ${ }^{2}$ Because of the absence of a defined organizational structure for dental sleep medicine clinics, respondents stated other challenges such as integrating into existing programs, administrative and billing issues etc. Similarly, a questionnaire-based study by Almohaya et al. ${ }^{37}$ identified two difficulties to increase the time for sleep medicine education: 53\% stated that the topic did not have high priority, whereas $47 \%$ indicated that there is insufficient time to teach the topic. In a study by Mindell et al., ${ }^{38} 12$ countries were surveyed and these difficulties were recorded: insufficient time, a lack of trained staff and resources, low priority, and irrelevance. It is alarming to note that sleep medicine did not get priority like other topics, and therefore, was understaffed with insufficient funding.

Interestingly, most respondents stated the presence of one to two board certified dental sleep medicine faculty members at their institution. This indicates a trend toward growing importance and presence of dental sleep medicine departments at academic institutions. Strategies such as increased collaboration among faculty members, and interdisciplinary approach between departments in the dental clinic may further aid in the development of a strong dental sleep medicine program.

Also, most respondents reported the presence of $\mathrm{CE}$ programs at their institution with courses being offered at least once a year, and show growing levels of involvement in dental sleep medicine practice and education at academic institutions. However, $60 \%$ of respondents $(6 / 10)$ thought that the courses offered were fewer than 25 hours. Hence, increasing the frequency and duration of $\mathrm{CE}$ courses on dental sleep medicine could further improve the dental sleep medicine practice. This study suggests that the emergence of mandibular advancement devices have contributed to the rise of dental sleep medicine practice. In line with those findings, many respondents stated that they offer a combination therapy of mandibular advancement device with either PAP or positional therapy to their patients.

The limitations of this study include being limited to the United States and Canada; with the electronic questionnaire other dental schools in Australia and Europe could 
Figure 5. The presence of continuing education programs in dental sleep medicine.

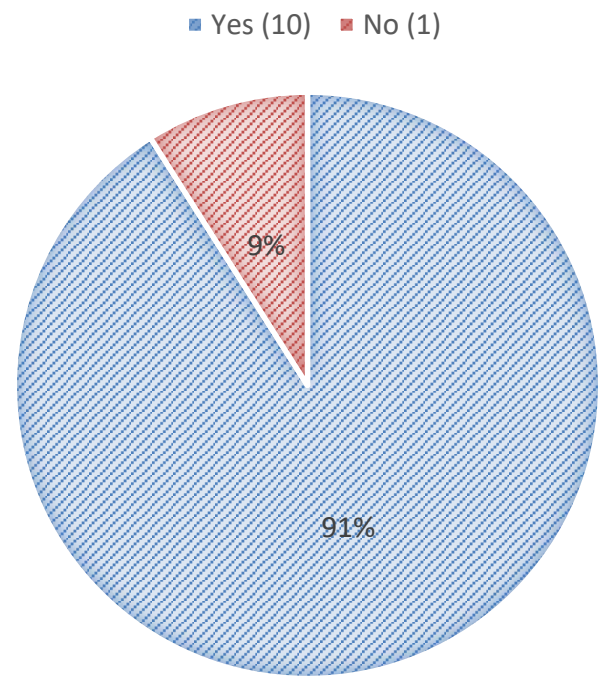

Figure 6. Frequency of sleep continuing education course offerings.

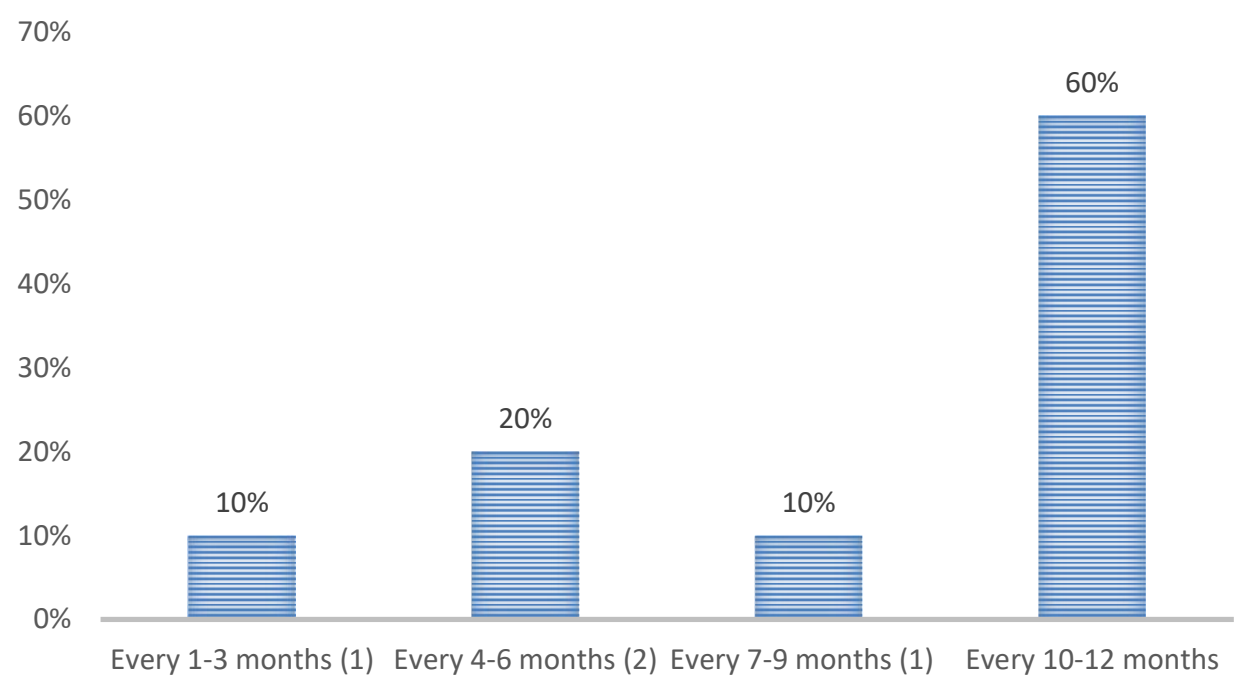

(6)

have been included. Another limitation was not supplementing the electronic questioner with a paper one, and that might have affected the response rate.

At this date this paper was written, the American Dental Education Association and the Council on Dental Accreditation had not created or implemented foundational educational protocols for Dental Sleep Medicine in the United States. Furthermore, incorporating more dental sleep medicine in the curriculum depends on the dean and faculty.

The results suggest that academic institutions consider dental sleep medicine an important topic to be included in their curriculums. In addition, respondents stated the presence of board certified dental sleep medicine faculty had a positive effect on their program. The study suggests that strategies such as increased collaboration among faculty members, and an interdisciplinary approach between departments in the dental clinic may further aid in the development of a strong dental sleep medicine program. As a result, the use of dental sleep medicine as a standard of care in dentistry should increase.

We speculate that with the recent development of dental sleep medicine standards of practice by the American Academy of Dental Sleep Medicine and the collaboration between academic institutions and professional associations, the practice and teaching of dental sleep medicine in academia will continue to grow globally.

\section{REFERENCES}

1. Young T, Finn L, Peppard PE, et al. Sleep disordered breathing and mortality: eighteen-year follow-up of the Wisconsin sleep cohort Sleep. 2008;31(8):1071-1078. 

development of insulin resistance and obesity. Endocrinol Metab Clin North Am. 2013;42(3):617-634.

3. Schillaci G, Battista F, Fiorenzano G, et al. Obstructive Sleep Apnea and Cardiovascular Disease - A New Target for Treatment. Curr Pharm Des. 2015;21(24):3496-3504.

4. Phillips B, Collop N, Goldberg R. Sleep medicine practices, training, and attitude: a wake-up call for pulmonologists. Chest. 2000;117(6):1603-1607.

5. BaHammam AS. Knowledge and attitude of primary health care physicians towards sleep disorders. Saudi Med J. 2000;21(12):11641167.

6. Kramer NR, Cook TE, Carlisle CC, Corwin RW, Millman PP. The role of the primary care physician in recognizing obstructive sleep apnea. Arch Intern Med. 1999;159(9):965-968.

7. Crisostomo I, Merritt S, Roe B, et al. Knowledge and attitudes regarding sleep medicine among first-year medical students, nursing students, and residents. Sleep. 1999; 22(suppl 1):S235-S236 27.

8. Kovacić Z, Marendić M, Soljić M, Pecotic R, Kardum G, Dogas Z. Knowledge and attitude regarding sleep medicine of medical students and physicians in Split, Croatia. Croat Med J. 2002;43(1):71-74.

9. Papp KK, Penrod CE, Strohl KP. Knowledge and attitude of primary care physicians toward sleep and sleep disorders. Sleep Breath. 2002;6(3):103-109.

10. Lin MC, Lee CF, Lin CL, et al. Non-apnea sleep disorder increases the risk of periodontal disease: a retrospective population-based cohort study. J Periodontol. 2014;85(4):e65-71.

11. Grover V, Malhotra R, Kaur H. Exploring association between sleep deprivation and chronic periodontitis: A pilot study. J Indian Soc Periodontol. 2015;19(3):304-307.

Yaggi HK, Strohl KP. Adult obstructive sleep apnea/ hypopnea syndrome: definitions, risk factors, and pathogenesis. Clin Chest Med. 2010;31(2):179-186

3. Kushida CA, Morgenthaler TI, Littner MR, et al. Practice parameters for oral appliances: an update for 2005 AASM practice parameters. Sleep. 2006;29(2):240-3.

14. Halberstadt J. Sleep Apnea: The Phantom Cause of Heart Disease and Accidents. New York, New York: New Technology Publishing, 2010.

15. Yaggi HK, Concato J, Kernan WN, Lichtman JH, Brass LM, Mohsenin V. Obstructive sleep apnea as a risk factor for stroke and death. N Engl J Med. 2005;353(19):2034-2041.

16. Garni AS, Pressman G, Caples SM, et al. Association of atrial fibrillation and obstructive sleep apnea. Circulation. 2004;110(4):364-367.

17. Kapur VK. Obstructive sleep apnea: diagnosis, epidemiology, and economics. Respir Care. 2010;55(9):1155-1167.

18. Drager LF, Bortolotto LA, Lorenzi MC, Fioueiredo AC, Krieger EM, Lorenzi-Filho G. Early signs of atherosclerosis in obstructive sleep apnea. Am J Respir Crit Care Med. 2005;172(5):613-618.

19. Mohsenin N, Mostofi MT, Mohsenin V. The role of oral appliances in treating obstructive sleep apnea. $J$ Am Dent Assoc. 2003;134(4):442-449.

20. Hossain JL, Shapiro CM. The prevalence, cost implications, and management of sleep disorders: an overview. Sleep Breath. 2002;6(2):85-102.

21. Institute of Medicine (US) Committee on Sleep Medicine and Research; Colten HR, Altevogt BM, editors. Sleep Disorders and Sleep Deprivation: An Unmet Public Health Problem. Washington, DC: National Academies Press, 2006.

22. Balasubramaniam R, Pullinger A, Simmons M. Sleep medicine education at dental schools in Australia and New Zealand. $J$ Dent Sleep Med. 2014;1(1):9-16.
Physician education in sleep and sleep disorders: a national survey of U.S. medical schools. Sleep. 1993;16(3):249-254.

24. Rosen RC, Mahowald M, Chesson A, et al. The Taskforce 2000 survey on medical education in sleep and sleep disorders. Sleep. 1998;2(3)1:235-238.

25. Ivanoff CS, Pancratz F. Incidence of Sleep Disorders Reported by Patients at UTHSC College of Dentistry: A Two-Year Follow-Up and Proposed Educational Program. J Dent Educ. 2015;79(5):548-556.

26. Talaat W, AlRozzi B, Kawas SA. Sleep medicine education and knowledge among undergraduate dental students in Middle East universities. Cranio. 2016;34(3):163-168.

27. Shahar E, Whitney CW, Redline S, et al. Sleep-disordered breathing and cardiovascular disease: Cross-sectional results of the Sleep Heart Health Study. Am J Respir Crit Care Med. 2001;163(1):19-25.

28. Franklin KA, Nilsson JB, Sahlin C, Näslund U. Sleep apnoea and nocturnal angina. Lancet. 1995;345(8957):1085-1087.

29. Mehra R, Benjamin EJ, Shahar E, et al. Association of nocturnal arrhythmias with sleep-disordered breathing: The Sleep Heart Health Study. Am J Respir Crit Care Med. 2006;173(8):910-916.

30. Ivanhoe JR, Frazier KB, Parr GR, Haywood VB. The teaching and treatment of upper airway sleep disorders in North American dental schools. J Prosthet Dent. 2003;89(3):292-296.

31. Bian H. Knowledge, opinions, and clinical experience of general practice dentists toward obstructive sleep apnea and oral appliances. Sleep Breath. 2004;8(2):85-90.

32. Ivanoff CS, Hottel TL, Pancratz F. Is there a place for teaching obstructive sleep apnea and snoring in the predoctoral dental curriculum? J Dent Educ. 2012;76(12):1639-1645.

33. Luyster FS, Strollo PJ Jr, Zee PC, Walsh JK; Boards of Directors of the American Academy of Sleep Medicine and the Sleep Research Society. Sleep: a health imperative. Sleep. 2012;35(6):727-734.

34. Chervin RD, Chesson AL Jr, Benca RM, et al. Organization and structure for sleep medicine programs at academic institutions: Part 1-current challenges. Sleep. 2013;36(6):795-801.

35. Loube MD, Strauss AM. Survey of oral appliance practice among dentists treating obstructive sleep apnea patients. Chest. 1997;111(2):382-386.

36. Simmons MS, Pullinger A. Education in sleep disorders in U.S. dental schools’ DDS programs. Sleep Breath. 2012;16(2):383-392.

37. Almohaya et al.: Sleep medicine education and knowledge among medical students in selected Saudi Medical Schools. BMC Med Educ. 2013 13:133.

38. Mindell JA, Bartle A, Ahn Y, et al: Sleep education in pediatric residency programs: a cross-cultural look. BMC Res Notes. $2013 ; 6: 130$

\section{SUBMISSION \& CORRESPONDENCE INFORMATION}

\section{Submitted for publication October 17, 2018 \\ Submitted in final revised form January 22, 2019 Accepted for publication February 3, 2019}

Address correspondence to: Leopoldo P. Correa, BDS, MS, Tufts University School of Dental Medicine, 1 Kneeland Street, Suite 601, Boston, MA, USA; Email: Leopoldo.correa@tufts.edu

\section{DISCLOSURE STATEMENT}

The authors have no conflicts of interest to disclose. 


\section{ABBREVIATIONS}

PAP: positive airway pressure

OSA: obstructive sleep apnea
SRBDs: sleep-related breathing disorders

CE: continuing education 


\section{APPENDIX}

\section{Survey}

The Current State of Dental Sleep Medicine Practice in Academic Institution: A Questionnaire-Based Study

Questions:

1. What country is your school in?
a. Canada
b. United States

2. What geographic region is your school in?
a. Northeast
b. Southeast
c. Midwest
d. Southwest
e. West

3. Is your university private or public?
a. Private
b. Public
c. Other (please specify)

4. Do you have a Dental Sleep Medicine Clinic at your institution?
a. Yes
b. No

5. Provide the name of the department where the clinic is incorporated
a. Orofacial pain and Temporomandibular Disorder
b. Endodontics
c. Orthodontics
d. Prosthodontics/ Preventive and Restorative Science
e. Pediatric Dentistry
f. Oral Surgery
g. Oral Medicine
h. Periodontics
i. Other (please specify) 
6. What challenges did you have implementing a dental sleep medicine clinic at your institution?
a. Administrative approval/support
b. Space for the clinic
c. Not a sufficient number of faculty members trained in dental sleep medicine
d. No challenges
e. Other (please specify)

7. How many board certified trained dental sleep medicine faculty members are at your institution?
a. 1 to 2
b. 3 to 5
c. more than 5

8. Who treats patients at the dental sleep medicine clinic in your institution?
a. Faculty only
b. Faculty and residents
c. Faculty, residents, and dental students

9. Where do you get your patient referral source? (select all that apply)
a. Sleep centers
b. Sleep physicians
c. Within the dental school (referral from other clinics)
d. Dentists
e. Other (please specify)

10. What type of oral appliance design do you use? (select all that apply)
a. Custom fabricated and adjustable
b. Prefabricated and adjustable
c. Temporary devices
d. Tongue retainer devices 
11. What type of combination therapy is offered to your patients? (select all that apply)
a. Mandibular advancement device and positive airway pressure (PAP) therapy
b. Tongue retaining device and PAP therapy
c. Mandibular advancement device and positional therapy
d. None

12. What type of technique do you use to minimize bite side effects? (select all that apply)
a. Morning (AM) aligner
b. Jaw exercises
c. Muscle stretching exercises
d. Morning repositioning device

13. When do you provide the technique from the previous question?
a. Provided at the time of sleep oral appliance delivery
b. As needed if patient develop side effects
c. Other (please specify)

14. What is your protocol schedule for follow-up visits?
a. Every 2 weeks
b. Every 4 weeks
c. Every 6 weeks
d. Other (please specify)

15. What is your schedule for long term follow ups?
a. 6 months and yearly after completion of therapy
b. As needed
c. Other (please specify)

16. How many patients are scheduled monthly?
a. 1 to 5
b. 5 to 15
c. 15 to 30
d. more than 30 
17. What percentage of patients are lost to follow up in your patient population?
a. Fewer than $10 \%$
b. $10 \%$ to $20 \%$
c. More than $20 \%$

18. What follow up method do you use to confirm treatment efficacy?
a. Polysomnography
b. Home Sleep Apnea Testing
c. Patient's feedback
d. Other (please specify)

19. Does your institution have continuing education programs in dental sleep medicine?
a) Yes
b) No

20. How often are sleep continuing education courses are offered?
a. Every 1 to 3 months
b. Every 4 to 6 months
c. Every 7 to 9 months
d. Every 10 to 12 months

21. Is your dental sleep continuing education course(s) program a minimum of 25 hours?
a. Yes
b. No 